\title{
Asymptotic Behavior of Solutions for Forest Kinematic Model
}

\author{
By
}

\author{
Le Huy Chuan ${ }^{1}$, Tohru Tsujikawa ${ }^{2}$ and Atsushi Yagi $^{3}$ \\ (Osaka University ${ }^{1,3}$ and Miyazaki University ${ }^{2}$, Japan)
}

\begin{abstract}
We continue a study of the mathematical model of forest ecosystem which has been introduced by Kuznetsov et al. [3]. In this paper, we will introduce three kinds of $\omega$-limit sets, namely, $\omega\left(U_{0}\right) \subset L^{2}-\omega\left(U_{0}\right) \subset \mathrm{w}^{*}-\omega\left(U_{0}\right)$, for each point $U_{0}$ of the dynamical system which was constructed in our preceding paper [1]. Using a Lyapunov function, we will then investigate basic properties of these $\omega$-limit sets. Especially it shall be shown that $L^{2}-\omega\left(U_{0}\right)$ consists of stationary solutions alone.

Key Words and Phrases. Asymptotic behavior of solutions, Dynamical system, Lyapunov function, $\omega$-limit set, Forestry ecosystem.

2000 Mathematics Subject Classification Numbers. 37L45, 37N25, 92D40.
\end{abstract}

\section{Introduction}

We study the initial-boundary value problem for a parabolic-ordinary system

$$
\begin{cases}\frac{\partial u}{\partial t}=\beta \delta w-\gamma(v) u-f u & \text { in } \Omega \times(0, \infty), \\ \frac{\partial v}{\partial t}=f u-h v & \text { in } \Omega \times(0, \infty), \\ \frac{\partial w}{\partial t}=d \Delta w-\beta w+\alpha v & \text { in } \Omega \times(0, \infty), \\ \frac{\partial w}{\partial n}=0 & \text { on } \partial \Omega \times(0, \infty), \\ u(x, 0)=u_{0}(x), v(x, 0)=v_{0}(x), w(x, 0)=w_{0}(x) & \text { in } \Omega .\end{cases}
$$

This system has been introduced by Kuznetsov et al. [3] in order to describe the development of a forest ecosystem. They considered an age-structured continuous model in a two-dimensional domain $\Omega$. For simplicity, they handle a prototype ecosystem of a mono-species which consists of only two age classes,

This work was supported by Grant-in-Aid for Scientific Research (No. 16340046) by Japan Society for the Promotion of Science and Cooperative Research Program in the form of Core University Program between JSPS and VAST (Vietnamese Academy of Science and Technology) by Japan Society for the Promotion of Science. 
namely, young age and old age. The unknown functions $u(x, t)$ and $v(x, t)$ denote the tree densities of young and old age classes, respectively, at a position $x \in \Omega$ and time $t \in[0, \infty)$. The third unknown function $w(x, t)$ denotes the density of seeds in the air at $x \in \Omega$ and $t \in[0, \infty)$. The third equation describes the kinetics of seeds; $d>0$ is a diffusion constant of seeds; and, $\alpha>0$ and $\beta>0$ are seed production and seed deposition rates, respectively. While the first and second equations describe the growth of young and old trees, respectively; $0<\delta \leq 1$ is a seed establishment rate; $\gamma(v)>0$ is a mortality of young trees which is allowed to depend on the old-tree density $v ; f>0$ is an aging rate; and, $h>0$ is a mortality of old trees. It is observed in general that $\gamma(v)$ has a minimum at some value of $v$, say $b$. A typical form of $\gamma(v)$ is given by

$$
\gamma(v)=a(v-b)^{2}+c
$$

where $a, b, c>0$ are positive constants, see [3]. In this paper, we assume that $\gamma(v)$ is given in this form and that $\Omega$ is a convex or $\mathscr{C}^{2}$, bounded domain in $\boldsymbol{R}^{2}$.

We treat this system as a degenerate parabolic system ('degenerate' means that the diffusion constants for $u$ and $v$ are considered to vanish identically). As we showed in the preceding paper [1], one can in fact construct the global solutions by regarding $(1.1)$ as the Cauchy problem for some appropriate abstract parabolic evolution equation in the product space

$$
X=\left\{\left(\begin{array}{c}
u \\
v \\
w
\end{array}\right) ; u, v \in L^{\infty}(\Omega) \text { and } w \in L^{2}(\Omega)\right\}
$$

for all initial values $U_{0}$ 's from the space

$$
K=\left\{U_{0}=\left(\begin{array}{c}
u_{0} \\
v_{0} \\
w_{0}
\end{array}\right) ; 0 \leq u_{0}, v_{0} \in L^{\infty}(\Omega) \text { and } 0 \leq w_{0} \in L^{2}(\Omega)\right\}
$$

(cf. Remark 2.1), and can also define a dynamical system $(S(t), K, X)$, cf. $[10,13,15]$ etc. Note that it is very natural to choose the space $L^{\infty}(\Omega)$ which is a Banach algebra $L^{\infty}(\Omega)$ under the usual function product (i.e., $\|u v\|_{L^{\infty}} \leq$ $\left.\|u\|_{L^{\infty}}\|v\|_{L^{\infty}}\right)$ as underlying spaces for formulating the first and second equations of (1.1) in view of the nonlinear function (1.2).

We encounter, however, a very unfavorable fact that our system does not always enjoy the smoothing effect of solutions. What is worse, as a numerical example presented in Section 6 (cf. also [6]) shows, we find in some case a solution which starts from continuous initial functions and converges asymptotically to a discontinuous stationary solution. For the moment, there 
is no rigorous analytical proof for this phenomenon, but we can certainly prove in such a case existence of the discontinuous stationary solutions, $u, v \in L^{\infty}(\Omega)$ being discontinuous and $w \in H^{2}(\Omega)$ being always continuous, see [2]. Such an unfavorable phenomenon is of course affected by the degeneracy of a diffusion for $u$ and the nonlinearity of the term $\gamma(v) u$. To clarify the situation more, it may be interesting to compare our system with another parabolic-ordinary system. For example, let us consider the Fitzhugh-Nagumo equations. As will be reviewed in Appendix, even if the initial data are discontinuous functions, the solution always has a time sequence $t_{n}$ tending to infinity for which $u\left(t_{n}\right)$ and $v\left(t_{n}\right)$ converge to smooth functions. In this sense, the Fitzhugh-Nagumo equations enjoy some weak smoothing effect. This is achieved by the difference that the ordinary equation of Fitzhugh-Nagumo is linear in contrast with (1.1).

In view of the forest ecosystem, such a solution with discontinuous density is rather expected one. The curve in $\Omega$ on which $u$ and $v$ have a discontinuous gap of density is called the ecotone boundary and is regarded as an internal boundary which the ecosystem possesses properly. To know how the ecotone boundary is formed by the parameters in (1.1) and to know how the ecotone boundary moves are one of main interests in the theory of mathematical forestry ecology, although they seem to be very hard problems.

In this paper, we are concerned with studying asymptotic behavior of solutions. As mentioned above, some solution starting from continuous initial functions may converge to discontinuous stationary solution; this then suggests that our dynamical system $(S(t), K, X)$ never possesses a global attractor in the topology of $X$. It is neither known to have a global attractor in any weak topology of $X$, for one cannot show continuity of the semigroup $S(t)$ in the weak* topology of $L^{\infty}(\Omega)$, although $S(t)$ enjoys a compact absorbing set in the weak* topology. In view of these situations, we are rather led to investigate asymptotic behavior of each trajectory of $(S(t), K, X)$. We will introduce three kinds of $\omega$-limit sets, namely, $\omega\left(U_{0}\right) \subset L^{2}-\omega\left(U_{0}\right) \subset \mathrm{w}^{*}-\omega\left(U_{0}\right)$ for $U_{0} \in K$. Here, $\omega\left(U_{0}\right)$ is the usual $\omega$-limit set in the topology of $X$ but may be empty for some $U_{0} \in K, L^{2}-\omega\left(U_{0}\right)$ is an $\omega$-limit set with respect to the $L^{2}$ topology, and $\mathrm{w}^{*}-\omega\left(U_{0}\right)$ is that with respect to the weak* topology of $L^{\infty}(\Omega)$. As shown by [1, Theorem 6.3], $\mathrm{w}^{*}-\omega\left(U_{0}\right)$ is always nonempty for every $U_{0} \in K$. Fortunately, we can find a Lyapunov function for our dynamical system. Owing the Lyapunov function, we can obtain many results on these $\omega$-limit sets. Among others, it is proved that $L^{2}-\omega\left(U_{0}\right)$ consists of stationary solutions alone. But, for the moment, it is an open problem to prove that $\mathrm{w}^{*}-\omega\left(U_{0}\right)$ consists of stationary solutions alone.

The next interesting problem may be to investigate the structure of stationary solutions. But the structure changes drastically with respect to the value of $h$. When $0<h<f \alpha \delta /\left(a b^{2}+c+f\right)$, it can be proved that stationary 
solutions are only homogeneous solutions in $\Omega$. As will be shown in Theorem 4.3, when $f \alpha \delta /(c+f)<h<\infty$, the zero solution $(0,0,0)$ is the only stationary solution of (1.1). To the contrary, when $f \alpha \delta /\left(a b^{2}+c+f\right)<h<f \alpha \delta /(c+f)$, the structure of stationary solutions, including possibly an infinite number of discontinuous ones, seems to be very complicated. This will be studied in the forthcoming paper [2].

For other results for $(1.1)$, we refer to $[4,8,9]$ in which a spatial average effect is incorporated.

Throughout the paper, $C$ stands for some constant which is determined by the initial constants $a, b, c, d, f, h, \alpha$ and $\beta$ and by the domain $\Omega$ in a specific way in each occurrence. So, it may change from occurrence to occurrence. Similarly, $p(\cdot)$ stands for some continuous increasing function which is determined in a specific way in each occurrence.

\section{Reviews}

In this section, we shall list some known results for (1.1) which have already been obtained in the previous paper [1], and shall also describe some consequences deduced from these which will be needed in the present paper.

The problem (1.1) is formulated as the Cauchy problem for an abstract evolution equation

$$
\left\{\begin{array}{l}
\frac{d U}{d t}+A U=F(U), \quad 0<t<\infty \\
U(0)=U_{0}
\end{array}\right.
$$

in the product space $X$. Here, $A$ is a sectorial operator of $X$ given by

$$
A=\left(\begin{array}{ccc}
f & 0 & 0 \\
0 & h & 0 \\
0 & 0 & \Lambda
\end{array}\right) \quad \text { with } \mathscr{D}(A)=\left\{\left(\begin{array}{c}
u \\
v \\
w
\end{array}\right) ; u, v \in L^{\infty}(\Omega) \text { and } w \in H_{N}^{2}(\Omega)\right\},
$$

where $\Lambda$ is a realization of the operator $-d \Delta+\beta$ in $L^{2}(\Omega)$ under the homogeneous Neumann boundary conditions $\partial w / \partial n=0$ on the boundary $\partial \Omega$. It is known that $\Lambda$ is a positive definite self-adjoint operator of $L^{2}(\Omega)$ with $\mathscr{D}(\Lambda)=$ $H_{N}^{2}(\Omega)$ (see $\left.[11,12]\right)$, where $H_{N}^{2}(\Omega)$ is a closed subspace of $H^{2}(\Omega)$ consisting of functions $w$ 's satisfying the homogeneous Neumann boundary conditions on $\partial \Omega$. It is also known that $\mathscr{D}\left(\Lambda^{\theta}\right)=H^{2 \theta}(\Omega)$ for $0 \leq \theta<3 / 4$ and $\mathscr{D}\left(\Lambda^{\theta}\right)=H_{N}^{2 \theta}(\Omega)$ for $3 / 4<\theta \leq 1$, where $H_{N}^{2 \theta}(\Omega)$ is a closed subspace of $H^{2 \theta}(\Omega)$ consisting of functions satisfying the homogeneous Neumann boundary conditions on $\partial \Omega$ (see $[5,16])$.

Meanwhile, $F$ is a nonlinear operator from $\mathscr{D}\left(A^{\eta}\right)$ into $X$ given by 


$$
F(U)=\left(\begin{array}{c}
\beta \delta w-\gamma(v) u \\
f u \\
\alpha v
\end{array}\right), \quad U=\left(\begin{array}{c}
u \\
v \\
w
\end{array}\right) \in \mathscr{D}\left(A^{\eta}\right),
$$

where $\eta$ is some fixed exponent such that $1 / 2<\eta<1$. Then, (1.1) is written in the form (2.1), see [1, Section 3].

According to [1] (especially Theorem 5.2), for any $U_{0} \in K,(2.1)$ possesses a unique global solution $U=(u, v, w)$ in the function space

$$
\begin{gathered}
0 \leq u, v \in \mathscr{C}\left([0, \infty) ; L^{\infty}(\Omega)\right) \cap \mathscr{C}^{1}\left((0, \infty) ; L^{\infty}(\Omega)\right), \\
0 \leq w \in \mathscr{C}\left((0, \infty) ; H_{N}^{2}(\Omega)\right) \cap \mathscr{C}\left([0, \infty) ; L^{2}(\Omega)\right) \cap \mathscr{C}^{1}\left((0, \infty) ; L^{2}(\Omega)\right) .
\end{gathered}
$$

Remark 2.1. In the statement of [1, Theorem 5.2], the functions $w_{0}$ 's in $U_{0}$ 's were assumed to be in $H^{2 \mu}(\Omega)$ with some $1 / 2<\mu<3 / 4$. But, actually, this restriction is not necessary, because, as [1, (3.3)] shows, it is possible to apply [7, Theorem 3.1] (namely, [1, Theorem 2.1]) to (2.1) with $\mu=0$ and $1 / 2<\eta<1$ (due to the fact that $\mathscr{D}\left(A^{\eta}\right) \subset L^{\infty}(\Omega)$ ). This means that it is allowed to take $w_{0}$ in such a way that $0 \leq w_{0} \in L^{2}(\Omega)$.

The solution satisfies the following integral equations (cf., $[1,(2.12)]$ ):

$$
\begin{gathered}
u(t)=e^{-\int_{0}^{t}\{\gamma(v(s))+f\} d s} u_{0}+\beta \delta \int_{0}^{t} e^{-\int_{s}^{t}\{\gamma(v(\tau))+f\} d \tau} w(s) d s, \quad 0 \leq t<\infty \\
v(t)=e^{-h t} v_{0}+f \int_{0}^{t} e^{-(t-s) h} u(s) d s, \quad 0 \leq t<\infty \\
w(t)=e^{-t \Lambda} w_{0}+\alpha \int_{0}^{t} e^{-(t-s) \Lambda} v(s) d s, \quad 0 \leq t<\infty
\end{gathered}
$$

Here, $e^{-t \Lambda}$ denotes the linear semigroup generated by $\Lambda$. Since $\Lambda \geq \beta$, it follows that $\left\|e^{-t \Lambda}\right\|_{L^{2}} \leq e^{-\beta t}$.

We verify the following uniform estimates of solutions which were essentially established in [1, Proposition 5.1].

Proposition 2.1. Let $U(t)=(u(t), v(t), w(t))$ be the global solution to (2.1) with $U_{0} \in K$. Then,

$$
\begin{array}{ll}
\|u(t)\|_{L^{\infty}} \leq p\left(\left\|U_{0}\right\|_{X}\right), & 0 \leq t<\infty, \\
\|v(t)\|_{L^{\infty}} \leq p\left(\left\|U_{0}\right\|_{X}\right), & 0 \leq t<\infty, \\
\|w(t)\|_{L^{2}} \leq p\left(\left\|U_{0}\right\|_{X}\right), & 0 \leq t<\infty,
\end{array}
$$

where $p(\cdot)$ denotes an appropriate continuous increasing function. 
Proof. We already know that

$$
\|U(t)\|_{L^{2}} \leq p\left(\left\|U_{0}\right\|_{L^{2}}\right), \quad 0 \leq t<\infty .
$$

From (2.6), it follows that

$$
\begin{aligned}
\|w(t)\|_{H^{2 \eta}} & \leq C\left\{\left\|\Lambda^{\eta} e^{-t \Lambda} w_{0}\right\|_{L^{2}}+\int_{0}^{t}\left\|\Lambda^{\eta} e^{-(t-s) \Lambda} v(s)\right\|_{L^{2}} d s\right\} \\
& \leq C\left(1+t^{-\eta}\right) e^{-\beta t}\left\|w_{0}\right\|_{L^{2}}+\int_{0}^{t}\left(1+(t-s)^{-\eta}\right) e^{-\beta(t-s)} d s p\left(\left\|U_{0}\right\|_{L^{2}}\right) \\
& \leq\left(1+t^{-\eta}\right) p\left(\left\|U_{0}\right\|_{L^{2}}\right), \quad 0<t<\infty .
\end{aligned}
$$

As $\|w(t)\|_{L^{\infty}} \leq C\|w(t)\|_{H^{2 n}}$, we obtain that

$$
\|w(t)\|_{L^{\infty}} \leq\left(1+t^{-\eta}\right) p\left(\left\|U_{0}\right\|_{L^{2}}\right), \quad 0<t<\infty .
$$

In view of the above inequality, we use (2.4) to obtain that $\|u(t)\|_{L^{\infty}} \leq\left\|u_{0}\right\|_{L^{\infty}}+\int_{0}^{t} e^{-f(t-s)}\left(1+s^{-\eta}\right) d s p\left(\left\|U_{0}\right\|_{L^{2}}\right) \leq p\left(\left\|U_{0}\right\|_{X}\right), \quad 0 \leq t<\infty$, i.e., (2.7). Similarly, (2.8) is obtained from (2.5) and (2.7).

In addition, we verify the uniform estimates for the derivative of solutions.

Proposition 2.2. For the derivative $U^{\prime}(t)=\left(u^{\prime}(t), v^{\prime}(t), w^{\prime}(t)\right)$,

$$
\begin{gathered}
\left\|u^{\prime}(t)\right\|_{L^{\infty}} \leq\left(1+t^{-\eta}\right) p_{1}\left(\left\|U_{0}\right\|_{X}\right), \quad 0<t<\infty, \\
\left\|v^{\prime}(t)\right\|_{L^{\infty}} \leq p_{1}\left(\left\|U_{0}\right\|_{X}\right), \quad 0<t<\infty, \\
\left\|w^{\prime}(t)\right\|_{L^{2}}+\|w(t)\|_{H^{2}} \leq\left(1+t^{-1}\right) p_{1}\left(\left\|U_{0}\right\|_{X}\right), \quad 0<t<\infty,
\end{gathered}
$$

where $p_{1}(\cdot)$ is an appropriate continuous increasing function.

Proof. Using (2.7), (2.8) and (2.10) in the equation on $u$ in (2.1), we immediately observe (2.11). Similarly, from the equation on $v$ in (2.1) we observe (2.12). We know that $v \in \mathscr{C}\left([0, \infty) ; L^{2}(\Omega)\right) \cap \mathscr{C}^{1}\left((0, \infty) ; L^{2}(\Omega)\right)$ with the estimate (2.12). Then, (2.13) is deduced by the standard arguments for the linear abstract equation on $w$ in (2.1), cf., (2.6).

We next obtain uniform estimates for the second order derivative of solutions.

Proposition 2.3. For the second order derivative $U^{\prime \prime}(t)=\left(u^{\prime \prime}(t), v^{\prime \prime}(t)\right.$, $\left.w^{\prime \prime}(t)\right)$,

$$
\left\|u^{\prime \prime}(t)\right\|_{L^{\infty}} \leq\left(1+t^{-1-\eta}\right) p_{2}\left(\left\|U_{0}\right\|_{X}\right), \quad 0<t<\infty,
$$




$$
\begin{gathered}
\left\|v^{\prime \prime}(t)\right\|_{L^{\infty}} \leq\left(1+t^{-\eta}\right) p_{2}\left(\left\|U_{0}\right\|_{X}\right), \quad 0<t<\infty, \\
\left\|w^{\prime \prime}(t)\right\|_{L^{2}}+\left\|w^{\prime}(t)\right\|_{H^{2}} \leq\left(1+t^{-2}\right) p_{2}\left(\left\|U_{0}\right\|_{X}\right), \quad 0<t<\infty,
\end{gathered}
$$

where $p_{2}(\cdot)$ is an appropriate continuous increasing function.

Proof. From the second equation in (2.1),

$$
v^{\prime \prime}(t)=f u^{\prime}(t)-h v^{\prime}(t), \quad 0<t<\infty .
$$

Then, $v \in \mathscr{C}^{2}\left((0, \infty) ; L^{\infty}(\Omega)\right)$ and the estimate $(2.15)$ is seen by $(2.11)$ and (2.12).

With any $\tau>0$, we consider the Cauchy problem for a linear evolution equation

$$
\left\{\begin{array}{l}
\frac{d w^{1}}{d t}+\Lambda w^{1}=\alpha v^{\prime}(t), \quad \tau<t<\infty, \\
w^{1}(\tau)=w^{\prime}(\tau)
\end{array}\right.
$$

in $L^{2}(\Omega)$, where $w^{1}=w^{1}(t)$ is the unknown function. Since $v^{\prime}$ is in $\mathscr{C}^{1}\left([\tau, \infty) ; L^{2}(\Omega)\right)$, this problem has a unique solution $w^{1} \in \mathscr{C}^{1}\left((\tau, \infty) ; L^{2}(\Omega)\right)$. By a direct calculation it is verified that $w^{1}(t)=w^{\prime}(t)$ for any $t \in[\tau, \infty)$. Therefore,

$$
w^{\prime}(t)=e^{-(t-\tau) \Lambda} w^{\prime}(\tau)+\alpha \int_{\tau}^{t} e^{-(t-s) \Lambda} v^{\prime}(s) d s, \quad \tau \leq t<\infty .
$$

Taking $\tau=t / 2$, we repeat the same argument as for (2.13) to obtain that

$$
\begin{aligned}
\left\|w^{\prime \prime}(t)\right\|_{L^{2}}+\left\|w^{\prime}(t)\right\|_{H^{2}} \leq & C\left(1+t^{-1}\right)\left\|w^{\prime}\left(\frac{t}{2}\right)\right\|_{L^{2}} \\
& +C\left\{p_{2}\left(\left\|U_{0}\right\|_{X}\right)+p_{1}\left(\left\|U_{0}\right\|_{X}\right)\right\}, \quad 0<t<\infty .
\end{aligned}
$$

Therefore, (2.16) is obtained in view of (2.13).

As a consequence of (2.13) and (2.16), we have

$$
\left\|w^{\prime}(t)\right\|_{L^{\infty}} \leq C\left\|w^{\prime}(t)\right\|_{H^{2 \eta}} \leq\left(1+t^{-1-\eta}\right) p\left(\left\|U_{0}\right\|_{X}\right), \quad 0<t<\infty .
$$

Then, (2.14) is observed directly from

$$
u^{\prime \prime}(t)=\beta \delta w^{\prime}(t)-\gamma^{\prime}(v(t)) v^{\prime}(t) u(t)-(\gamma(v(t))+f) u^{\prime}(t), \quad 0<t<\infty .
$$

We conclude this section with describing the dynamical system determined by the Cauchy problem (2.1). For any $U_{0} \in K$, let $U\left(t ; U_{0}\right)$ be the global solution of (2.1). We set $S(t) U_{0}=U\left(t ; U_{0}\right)$ for every $0 \leq t<\infty$. Then $S(t)$ defines a nonlinear semigroup acting on $K$. According to [1, Proposition 5.3], this semigroup is continuous on $K$ in the sense that the mapping $\left(t, U_{0}\right) \in$ $[0, \infty) \times K \rightarrow K$ is continuous. Therefore, the set of all trajectories $S(t) U_{0}$ 's 
defines a dynamical system in $X$ with phase space $K$ which is denoted by $(S(t), K, X)$.

According to [1, Theorem 6.1], there exists an invariant and absorbing set $\mathscr{X}$ for $S(t)$ which is a bounded set of $\mathscr{D}(A)$, namely,

$$
\begin{gathered}
\mathscr{X} \subset\left\{\left(\begin{array}{c}
u \\
v \\
w
\end{array}\right) ; 0 \leq u, v \in L^{\infty}(\Omega) \text { and } 0 \leq w \in H_{N}^{2}(\Omega)\right. \\
\text { with } \left.\|u\|_{L^{\infty}}+\|v\|_{L^{\infty}}+\|w\|_{H^{2}} \leq C_{\mathscr{X}}\right\}
\end{gathered}
$$

with some constant $0<C_{\mathscr{X}}<\infty$. Therefore, $(S(t), \mathscr{X}, X)$ is also a dynamical system and the asymptotic behavior of trajectories of $(S(t), K, X)$ is reduced to that of $(S(t), \mathscr{X}, X)$.

\section{Lyapunov function}

In this section we shall construct a Lyapunov function $\Psi(U)$ for the dynamical system $(S(t), K, X)$ and shall establish some results concerning the asymptotic behavior of trajectories $S(t) U_{0}$ 's.

Let $U_{0} \in K$ and let $S(t) U_{0}=U(t)=(u(t), v(t), w(t))$ for $0 \leq t<\infty$. Set $\varphi(t)=f u(t)-h v(t), 0 \leq t<\infty$. From the first and second equations of (1.1) it is easily observed that

$$
\frac{\partial \varphi}{\partial t}=f \beta \delta w-\{\gamma(v)+f+h\} \varphi-h\{\gamma(v) v+f v\}, \quad 0<t<\infty .
$$

Multiply this by $\varphi(t)=\partial v / \partial t$ and integrate the product in $\Omega$. Then,

$$
\begin{gathered}
\frac{1}{2} \frac{d}{d t} \int_{\Omega} \varphi^{2} d x+h \frac{d}{d t} \int_{\Omega} \Gamma(v) d x-f \beta \delta \int_{\Omega} \frac{\partial v}{\partial t} w d x \\
=-\int_{\Omega}\{\gamma(v)+f+h\}\left(\frac{\partial v}{\partial t}\right)^{2} d x,
\end{gathered}
$$

where $\Gamma(v)=\int_{0}^{v}\{\gamma(v) v+f v\} d v$.

While, multiplying the third equation of (1.1) by $\partial w / \partial t$ and integrating the product in $\Omega$, we obtain that

$$
\frac{d}{2} \frac{d}{d t} \int_{\Omega}|\nabla w|^{2} d x+\frac{\beta}{2} \frac{d}{d t} \int_{\Omega} w^{2} d x-\alpha \int_{\Omega} v \frac{\partial w}{\partial t} d x=-\int_{\Omega}\left(\frac{\partial w}{\partial t}\right)^{2} d x
$$

These two energy equalities (3.1) and (3.2) then provide that 


$$
\begin{aligned}
\frac{d}{d t} \int_{\Omega} & {\left[\frac{\alpha}{2} \varphi^{2}+\frac{d f \beta \delta}{2}|\nabla w|^{2}+h \alpha \Gamma(v)+\frac{f \beta^{2} \delta}{2} w^{2}-(f \alpha \beta \delta) v w\right] d x } \\
\quad & =-\int_{\Omega}\left[\alpha\{\gamma(v)+f+h\}\left(\frac{\partial v}{\partial t}\right)^{2}+f \beta \delta\left(\frac{\partial w}{\partial t}\right)^{2}\right] d x \leq 0, \quad 0<t<\infty
\end{aligned}
$$

Note that

$$
\frac{\alpha}{2}(f u-h v)^{2}+\frac{d f \beta \delta}{2}|\nabla w|^{2}+h \alpha \Gamma(v)+\frac{f \beta^{2} \delta}{2} w^{2}-(f \alpha \beta \delta) v w \geq C
$$

with some constant $C$ independent of $U$. This shows that the functional

$$
\begin{aligned}
\Psi(U)=\int_{\Omega} & {\left[\frac{\alpha}{2}(f u-h v)^{2}+\frac{d f \beta \delta}{2}|\nabla w|^{2}+h \alpha \Gamma(v)\right.} \\
& \left.+\frac{f \beta^{2} \delta}{2} w^{2}-(f \alpha \beta \delta) v w\right] d x, \quad U \in \mathscr{D}\left(A^{1 / 2}\right)
\end{aligned}
$$

is a Lyapunov function for the present dynamical system $(S(t), K, X)$.

From these arguments we obtain the following energy estimates.

Theorem 3.1. For any trajectory $S(t) U_{0}=U(t)$, we have

$$
\int_{1}^{\infty}\left\|\frac{d U}{d t}(t)\right\|_{L^{2}}^{2} d t<\infty
$$

Proof. Integrate both the sides of (3.3) in $t$ on an interval $[1, T]$. Then,

$$
\begin{aligned}
& \int_{1}^{T} \int_{\Omega}\left[\alpha\{\gamma(v)+f+h\}\left(\frac{\partial v}{\partial t}\right)^{2}+f \beta \delta\left(\frac{\partial w}{\partial t}\right)^{2}\right] d x d t \\
& \quad \leq \int_{\Omega}\left[\frac{\alpha}{2} \varphi(1)^{2}+\frac{d f \beta \delta}{2}|\nabla w(1)|^{2}+h \alpha \Gamma(v(1))+\frac{f \beta^{2} \delta}{2} w(1)^{2}+f \alpha \beta \delta v(T) w(T)\right] d x .
\end{aligned}
$$

Due to (2.8), (2.10), (2.12) and (2.13)

$$
\int_{1}^{\infty} \int_{\Omega}\left[\alpha\{\gamma(v)+f+h\}\left(\frac{\partial v}{\partial t}\right)^{2}+f \beta \delta\left(\frac{\partial w}{\partial t}\right)^{2}\right] d x d t<\infty .
$$

Differentiating both the sides of the first equation of (1.1), we have

$$
\frac{\partial^{2} u}{\partial t^{2}}=\beta \delta \frac{\partial w}{\partial t}-(\gamma(v)+f) \frac{\partial u}{\partial t}-2 a u(v-b) \frac{\partial v}{\partial t}, \quad 0<t<\infty .
$$

Multiply this by $\partial u / \partial t$ and integrate the product in $\Omega$. Then, 


$$
\begin{aligned}
\frac{1}{2} \frac{d}{d t} \int_{\Omega}\left(\frac{\partial u}{\partial t}\right)^{2} d x & =\int_{\Omega}\left(\beta \delta \frac{\partial w}{\partial t}-2 a u(v-b) \frac{\partial v}{\partial t}\right) \frac{\partial u}{\partial t} d x-\int_{\Omega}(\gamma(v)+f)\left(\frac{\partial u}{\partial t}\right)^{2} d x \\
& \leq p\left(\left\|U_{0}\right\|_{X}\right) \int_{\Omega}\left(\left|\frac{\partial v}{\partial t}\right|+\left|\frac{\partial w}{\partial t}\right|\right)\left|\frac{\partial u}{\partial t}\right| d x-f \int_{\Omega}\left(\frac{\partial u}{\partial t}\right)^{2} d x
\end{aligned}
$$

Therefore,

$$
\frac{1}{2} \frac{d}{d t} \int_{\Omega}\left(\frac{\partial u}{\partial t}\right)^{2} d x+\frac{f}{2} \int_{\Omega}\left(\frac{\partial u}{\partial t}\right)^{2} d x \leq p\left(\left\|U_{0}\right\|_{X}\right) \int_{\Omega}\left\{\left(\frac{\partial v}{\partial t}\right)^{2}+\left(\frac{\partial w}{\partial t}\right)^{2}\right\} d x
$$

Integrating both the sides in $t$ on an interval $[1, T]$, we obtain that

$$
\begin{aligned}
\frac{f}{2} \int_{1}^{T} \int_{\Omega}\left(\frac{\partial u}{\partial t}\right)^{2} d x d t \leq & \frac{1}{2} \int_{\Omega}\left(\frac{\partial u}{\partial t}(1)\right)^{2} d x \\
& +p\left(\left\|U_{0}\right\|_{X}\right) \int_{1}^{T} \int_{\Omega}\left\{\left(\frac{\partial v}{\partial t}\right)^{2}+\left(\frac{\partial w}{\partial t}\right)^{2}\right\} d x d t
\end{aligned}
$$

Hence, in view of (3.6), we conclude that

$$
\int_{1}^{\infty} \int_{\Omega}\left(\frac{\partial u}{\partial t}\right)^{2} d x d t<\infty
$$

This together with (3.6) then yields the desired estimate (3.5).

Theorem 3.2. For any trajectory $S(t) U_{0}=U(t)$, as $t \rightarrow \infty$, the derivative $(d U / d t)(t)$ tends to 0 in the $L^{2}$ topology.

Proof. We prove the assertion of theorem by contradiction. Suppose that $(d U / d t)(t)$ might not converge to 0 in $L^{2}(\Omega)$ as $t \rightarrow \infty$. Then there would exist a number $\varepsilon>0$ and a time sequence $\left\{t_{n}\right\}$ tending to $\infty$ such that

$$
\left\|\frac{d U}{d t}\left(t_{n}\right)\right\|_{L^{2}}^{2} \geq \varepsilon, \quad n=1,2,3, \ldots
$$

In the meantime, by Propositions 2.2 and 2.3, we have

$$
\left|\frac{d}{d t}\left\|\frac{d U}{d t}(t)\right\|_{L^{2}}^{2}\right|=2\left|\left(\frac{d^{2} U}{d t^{2}}(t), \frac{d U}{d t}(t)\right)_{L^{2}}\right| \leq M, \quad 1 \leq t<\infty
$$

with some constant $M$. Consequently, by the mean-value theorem,

$$
\left\|\frac{d U}{d t}(t)\right\|_{L^{2}}^{2} \geq \begin{cases}M\left(t-t_{n}+\frac{\varepsilon}{M}\right), & t_{n}-\frac{\varepsilon}{M} \leq t \leq t_{n} \\ -M\left(t-t_{n}-\frac{\varepsilon}{M}\right), & t_{n} \leq t \leq t_{n}+\frac{\varepsilon}{M}\end{cases}
$$


This is a contradiction to the fact that $\|(d U / d t)(t)\|_{L^{2}}^{2}$ is integrable in $(1, \infty)$, i.e., (3.5).

\section{4. $\omega$-limit sets}

In this section, we shall introduce three types of $\omega$-limit sets, namely, $\omega\left(U_{0}\right), L^{2}-\omega\left(U_{0}\right)$ and $\mathrm{w}^{*}-\omega\left(U_{0}\right)$, and shall investigate their relations.

As well known, the (usual) $\omega$-limit set of $S(t) U_{0}, U_{0} \in K$, is defined by

$$
\omega\left(U_{0}\right)=\bigcap_{t \geq 0} \overline{\left\{S(\tau) U_{0} ; t \leq \tau<\infty\right\}} \quad \text { (closure in the topology of } X \text { ), }
$$

namely, $\bar{U} \in \omega\left(U_{0}\right)$ if and only if there exists a time sequence $\left\{t_{n}\right\}$ tending to $\infty$ such that $S\left(t_{n}\right) U_{0} \rightarrow \bar{U}$ in the topology of $X$. As will be presented in Section 6, some numerical simulation suggests that there exists a trajectory which starts from a continuous initial function $U_{0}=\left(u_{0}(x), v_{0}(x), w_{0}(x)\right) \in K$ but, as $t \rightarrow \infty$, converges to a discontinuous stationary solution $\bar{U}=(\bar{u}(x), \bar{v}(x), \bar{w}(x))$. If this phenomenon is true, then any sequence $S\left(t_{n}\right) U_{0}$ cannot converge to $\bar{U}$ in the topology of $X$, namely, it is possible that $\omega\left(U_{0}\right)=\varnothing$.

We define the $L^{2}$ topology of $X$ as follows. A sequence $\left\{\left(u_{n}, v_{n}, w_{n}\right)\right\}$ in $X$ is said to be $L^{2}$ convergent to $\left(u_{0}, v_{0}, w_{0}\right) \in X$ as $n \rightarrow \infty$, if

$$
\begin{cases}u_{n} \rightarrow u_{0} & \text { strongly in } L^{2}(\Omega), \\ v_{n} \rightarrow v_{0} & \text { strongly in } L^{2}(\Omega), \\ w_{n} \rightarrow w_{0} & \text { strongly in } L^{2}(\Omega) .\end{cases}
$$

Then, using this topology we define the $L^{2}$ - $\omega$-limit set of $S(t) U_{0}, U_{0} \in K$, by

$$
L^{2}-\omega\left(U_{0}\right)=\bigcap_{t \geq 0} \overline{\left\{S(\tau) U_{0} ; t \leq \tau<\infty\right\}} \quad\left(\text { closure in the } L^{2} \text { topology of } X\right) .
$$

In addition, we may equip $X$ with the weak* topology. A sequence $\left\{\left(u_{n}, v_{n}, w_{n}\right)\right\}$ in $X$ is said to be weak* convergent to $\left(u_{0}, v_{0}, w_{0}\right) \in X$ as $n \rightarrow \infty$, if

$$
\begin{cases}u_{n} \rightarrow u_{0} & \text { weak }^{*} \text { in } L^{\infty}(\Omega), \\ v_{n} \rightarrow v_{0} & \text { weak }^{*} \text { in } L^{\infty}(\Omega), \\ w_{n} \rightarrow w_{0} & \text { strongly in } L^{2}(\Omega) .\end{cases}
$$

Using this topology, we define the $\mathrm{w}^{*}-\omega$-limit set of $S(t) U_{0}, U_{0} \in K$, by

$$
\left.\mathrm{w}^{*}-\omega\left(U_{0}\right)=\bigcap_{t \geq 0} \overline{\left\{S(\tau) U_{0} ; t \leq \tau<\infty\right\}} \quad \text { (closure in the weak }{ }^{*} \text { topology of } X\right) .
$$

According to [1, Theorem 6.3], it is already known that $\mathrm{w}^{*}-\omega\left(U_{0}\right) \neq \varnothing$ for any initial data $U_{0} \in K$.

In general we observe the following relations. 
Theorem 4.1. For each $U_{0} \in K, \omega\left(U_{0}\right) \subset L^{2}-\omega\left(U_{0}\right) \subset \mathrm{w}^{*}-\omega\left(U_{0}\right)$.

Proof. The first relation $\omega\left(U_{0}\right) \subset L^{2}-\omega\left(U_{0}\right)$ is obvious by the definition.

Let $\bar{U}=(\bar{u}, \bar{v}, \bar{w}) \in L^{2}-\omega\left(U_{0}\right)$. Then, there exists a sequence $\left\{t_{n}\right\}$ tending to $\infty$ such that $S\left(t_{n}\right) U_{0}=\left(u\left(t_{n}\right), v\left(t_{n}\right), w\left(t_{n}\right)\right) \rightarrow \bar{U}$ in the $L^{2}$ topology of $X$. Let $\varphi \in L^{1}(\Omega)$. For any $f \in L^{2}(\Omega)$,

$$
\left|\int_{\Omega} \varphi\left\{u\left(t_{n}\right)-\bar{u}\right\} d x\right| \leq\|\varphi-f\|_{L^{1}}\left\|u\left(t_{n}\right)-\bar{u}\right\|_{L^{\infty}}+\left|\int_{\Omega} f\left\{u\left(t_{n}\right)-\bar{u}\right\} d x\right| .
$$

Since $L^{2}(\Omega)$ is dense in $L^{1}(\Omega)$ and since (2.7) is valid, we verify that, as $t_{n} \rightarrow \infty$,

$$
\left|\int_{\Omega} \varphi\left\{u\left(t_{n}\right)-\bar{u}\right\} d x\right| \rightarrow 0 .
$$

Hence, $u\left(t_{n}\right) \rightarrow \bar{u}$ in the weak* topology of $L^{\infty}(\Omega)$. Due to (2.8), it is the same for the weak* convergence of $v\left(t_{n}\right)$ to $\bar{v}$. Thus we have $\bar{U} \in \mathrm{w}^{*}-\omega\left(U_{0}\right)$.

We do not know whether the converse relation $\mathrm{w}^{*}-\omega\left(U_{0}\right) \subset L^{2}-\omega\left(U_{0}\right)$ is true in general or not. We can however prove some weak result.

Theorem 4.2. For $U_{0} \in K$, let there exist a sequence $\left\{t_{n}\right\}$ tending to $\infty$ such that $S\left(t_{n}\right) U_{0}=\left(u\left(t_{n}\right), v\left(t_{n}\right), w\left(t_{n}\right)\right)$ converges to a triplet of functions $\bar{U}=$ $(\bar{u}, \bar{v}, \bar{w}) \in X$ almost everywhere in $\Omega$. Then, $\bar{U} \in L^{2}-\omega\left(U_{0}\right)$.

Proof. By virtue of (2.7), (2.8) and (2.10), the almost everywhere convergence implies $L^{2}$ convergence for each sequence of $u\left(t_{n}\right), v\left(t_{n}\right)$ and $w\left(t_{n}\right)$. Hence, $\bar{U} \in L^{2}-\omega\left(U_{0}\right)$.

The rest of this section is devoted to proving some structural results for the $\omega$-limit sets under specific conditions assumed to hold for the coefficients of equations in (1.1).

Theorem 4.3. Assume that $h>f \alpha \delta /(c+f)$. Then, $\omega\left(U_{0}\right)=L^{2}-\omega\left(U_{0}\right)$ $=\mathrm{w}^{*}-\omega\left(U_{0}\right)=\{(0,0,0)\}$ for every $U_{0} \in K$.

Proof. Let $U_{0}=\left(u_{0}, v_{0}, w_{0}\right) \in K$ and let $S(t) U_{0}=(u(t), v(t), w(t))$ be the global solution. Multiply the first equation of $(1.1)$ by $2(c+f) u$ and integrate the product in $\Omega$. Then,

$$
\begin{gathered}
(c+f) \frac{d}{d t} \int_{\Omega} u^{2} d x+2(c+f)^{2} \int_{\Omega} u^{2} d x-2(c+f) \beta \delta \int_{\Omega} w u d x \\
=-2 a(c+f) \int_{\Omega}(v-b)^{2} u^{2} d x \leq 0, \quad 0<t<\infty .
\end{gathered}
$$

Similarly, multiply the second equation of $(1.1)$ by $(2(c+f) \alpha \delta / f) v$ and integrate the product in $\Omega$. Then, 


$$
\begin{gathered}
\frac{(c+f) \alpha \delta}{f} \frac{d}{d t} \int_{\Omega} v^{2} d x+2(\alpha \delta)^{2} \int_{\Omega} v^{2} d x-2(c+f) \alpha \delta \int_{\Omega} u v d x \\
+\frac{2(c+f) \alpha \delta}{f}\left(h-\frac{f \alpha \delta}{c+f}\right) \int_{\Omega} v^{2} d x=0, \quad 0<t<\infty .
\end{gathered}
$$

Multiply the third equation of (1.1) by $2 \beta \delta^{2} w$ and integrate the product in $\Omega$. Then,

$$
\begin{gathered}
\beta \delta^{2} \frac{d}{d t} \int_{\Omega} w^{2} d x+2(\beta \delta)^{2} \int_{\Omega} w^{2} d x-2 \alpha \beta \delta^{2} \int_{\Omega} v w d x \\
\quad=-2 d \beta \delta^{2} \int_{\Omega}|\nabla w|^{2} d x \leq 0, \quad 0<t<\infty
\end{gathered}
$$

Summing up (4.1), (4.2) and (4.3), we obtain that

$$
\begin{aligned}
& \frac{d}{d t} \int_{\Omega}\left((c+f) u^{2}+\frac{(c+f) \alpha \delta}{f} v^{2}+\beta \delta^{2} w^{2}\right) d x \\
& \quad+2 \int_{\Omega}\left\{((c+f) u)^{2}+(\alpha \delta v)^{2}+(\beta \delta w)^{2}\right\} d x \\
& \quad-2 \int_{\Omega}\{(c+f) u \alpha \delta v+\alpha \delta v \beta \delta w+\beta \delta w(c+f) u\} d x+3 \int_{\Omega} \varepsilon v^{2} d x \leq 0
\end{aligned}
$$

where $\varepsilon=2(c+f) \alpha \delta(h-(f \alpha \delta /(c+f))) /(3 f)>0$. We here notice that

$$
\begin{aligned}
2\left\{((c+f) u)^{2}+(\alpha \delta v)^{2}+(\beta \delta w)^{2}-(c+f) u \alpha \delta v-\alpha \delta v \beta \delta w-\beta \delta w(c+f) u\right\}+3 \varepsilon v^{2} \\
=\left\{\frac{((c+f) \alpha \delta)^{2}}{\alpha^{2} \delta^{2}+\varepsilon} u^{2}-2(c+f) u \alpha \delta v+\left(\alpha^{2} \delta^{2}+\varepsilon\right) v^{2}\right\} \\
+\left\{\left(\alpha^{2} \delta^{2}+\varepsilon\right) v^{2}-2 \alpha \delta v \beta \delta w+\frac{(\alpha \delta)^{2}(\beta \delta)^{2}}{\alpha^{2} \delta^{2}+\varepsilon} w^{2}\right\}+\{\beta \delta w-(c+f) u\}^{2} \\
+\varepsilon\left\{\frac{(c+f)^{2}}{\alpha^{2} \delta^{2}+\varepsilon} u^{2}+v^{2}+\frac{(\beta \delta)^{2}}{\alpha^{2} \delta^{2}+\varepsilon} w^{2}\right\} .
\end{aligned}
$$

Therefore, with an appropriate exponent $\rho>0$ and appropriate constants $C_{i}>0, i=1,2,3$,

$$
\frac{d}{d t} \int_{\Omega}\left(C_{1} u^{2}+C_{2} v^{2}+C_{3} w^{2}\right) d x+\rho \int_{\Omega}\left(C_{1} u^{2}+C_{2} v^{2}+C_{3} w^{2}\right) d x \leq 0 .
$$

We thus conclude that

$$
\begin{aligned}
& C_{1}\|u(t)\|_{L^{2}}^{2}+C_{2}\|v(t)\|_{L^{2}}^{2}+C_{3}\|w(t)\|_{L^{2}}^{2} \\
& \quad \leq e^{-\rho t}\left(C_{1}\left\|u_{0}\right\|_{L^{2}}^{2}+C_{2}\left\|v_{0}\right\|_{L^{2}}^{2}+C_{3}\left\|w_{0}\right\|_{L^{2}}^{2}\right), \quad 0<t<\infty .
\end{aligned}
$$


As a result, as $t \rightarrow \infty, S(t) U_{0}$ converges to $(0,0,0)$ in the $L^{2}$ topology. More strongly, since $\|w(t)\|_{L^{\infty}} \leq C_{\varepsilon}\|w(t)\|_{H^{1+\varepsilon}} \leq C_{\varepsilon}\|w(t)\|_{L^{2}}^{(1-\varepsilon) / 2}\|w(t)\|_{H^{2}}^{(1+\varepsilon) / 2}$, we deduce from the $L^{2}$ convergence of $w(t)$ that in the $L^{\infty}$ topology (due to (2.13)). Furthermore, from the formulae (2.4) and (2.5), this implies convergence of $u(t)$ and $v(t)$ to 0 in the $L^{\infty}$ topology. In this way, we ultimately conclude that, as $t \rightarrow \infty, S(t) U_{0}$ converges to $(0,0,0)$ in the $L^{\infty}$ topology. From this the assertion of theorem follows immediately.

Theorem 4.4. Assume that $a b^{2}<3(c+f)$. Then, $L^{2}-\omega\left(U_{0}\right)=\mathrm{w}^{*}-\omega\left(U_{0}\right)$ for every $U_{0} \in K$.

Proof. Let $S(t) U_{0}=U(t)=(u(t), v(t), w(t))$. Consider any time sequence $\left\{t_{n}\right\}$ which tends to $\infty$ as $n \rightarrow \infty$. By (2.9), $\left\|w\left(t_{n}\right)\right\|_{H^{2}}$ is a bounded sequence; so, we can choose a subsequence $\left\{t_{n^{\prime}}\right\}$ for which $\left\{w\left(t_{n^{\prime}}\right)\right\}$ is convergent to $\bar{w}$ in $H^{1+\varepsilon}(\Omega)$ and hence in $L^{\infty}(\Omega)$. From the first and second equations of $(2.1)$ it is easily observed that

$$
\left(\gamma\left(v\left(t_{n^{\prime}}\right)\right)+f\right) v\left(t_{n^{\prime}}\right)=\frac{f}{h}\left\{\beta \delta w\left(t_{n^{\prime}}\right)-\frac{d u}{d t}\left(t_{n^{\prime}}\right)-\frac{\gamma\left(v\left(t_{n^{\prime}}\right)\right)+f}{f} \frac{d v}{d t}\left(t_{n^{\prime}}\right)\right\} .
$$

Here, we introduce the cubic function

$$
P(v) \equiv(\gamma(v)+f) v=a v^{3}-2 a b v^{2}+\left(a b^{2}+c+f\right) v, \quad-\infty<v<\infty .
$$

It is easy to see the following property.

Lemma 4.5. When $a b^{2}<3(c+f), w=P(v)$ is a monotone increasing function for $v \in(-\infty, \infty)$. Its inverse function $P^{-1}(w)$ is a single-valued smooth function for $w$ with uniformly bounded derivative in the whole real axis $w \in(-\infty, \infty)$.

Proof of Lemma. Obviously we have

$$
P^{\prime}(v)=3 a v^{2}-4 a b v+\left(a b^{2}+c+f\right)=3 a\left(v-\frac{2 b}{3}\right)^{2}-\frac{a b^{2}-3(c+f)}{3}>0 .
$$

Therefore, the assertion of lemma is clear.

Using $P^{-1}(w)$, we can write

$$
v\left(t_{n^{\prime}}\right)=P^{-1}\left(\frac{f}{h}\left\{\beta \delta w\left(t_{n^{\prime}}\right)-\frac{d u}{d t}\left(t_{n^{\prime}}\right)-\frac{\gamma\left(v\left(t_{n^{\prime}}\right)\right)+f}{f} \frac{d v}{d t}\left(t_{n^{\prime}}\right)\right\}\right) .
$$

Since $w\left(t_{n^{\prime}}\right) \rightarrow \bar{w}$ in $L^{\infty}(\Omega)$ and since Theorem 3.2 is true, we conclude that $v\left(t_{n^{\prime}}\right)$ converges to $\bar{v}=P^{-1}((f \beta \delta / h) \bar{w})$ in $L^{2}(\Omega)$. Since Theorem 3.2 provides in particular that, as $t \rightarrow \infty, f u(t)-h v(t) \rightarrow 0$ in $L^{2}(\Omega)$, we conclude also that $u\left(t_{n^{\prime}}\right)$ converges to $(h / f) \bar{v}$ in $L^{2}(\Omega)$. Thus we have shown that $\left(u\left(t_{n^{\prime}}\right), v\left(t_{n^{\prime}}\right), w\left(t_{n^{\prime}}\right)\right) \rightarrow(\bar{u}, \bar{v}, \bar{w})$ in $L^{2}(\Omega)$. 
We now know that any sequence $\left(u\left(t_{n}\right), v\left(t_{n}\right), w\left(t_{n}\right)\right)$ has a subsequence which converges to some vector of $X$ in the $L^{2}$ topology. Hence, the relation $\mathrm{w}^{*}-\omega\left(U_{0}\right) \subset L^{2}-\omega\left(U_{0}\right)$ is proved, cf., Proof of Theorem 4.1.

\section{Constituents of $L^{2}$ - $\omega$-limit sets}

In this the section, we shall show that every $L^{2}-\omega$-limit set consists of stationary solutions of (2.1). We begin with verifying the following proposition.

Proposition 5.1. For each $U_{0} \in K, L^{2}-\omega\left(U_{0}\right)$ is an invariant set of $S(t)$, i.e.,

$$
S(t)\left(L^{2}-\omega\left(U_{0}\right)\right) \subset L^{2}-\omega\left(U_{0}\right), \quad 0<t<\infty .
$$

Proof. In the proof of this proposition, it is essential to show that $S(t)$ is continuous from $K$ into itself in the $L^{2}$ topology.

To see this, consider two initial values $U_{01}=\left(u_{01}, v_{01}, w_{01}\right)$ and $U_{02}=$ $\left(u_{02}, v_{02}, w_{02}\right)$ in $K$, and let $\left(u_{1}(t), v_{1}(t), w_{1}(t)\right)$ and $\left(u_{2}(t), v_{2}(t), w_{2}(t)\right)$ be the solutions to $(2.1)$ with the initial value $U_{01}$ and $U_{02}$, respectively. Let $T>0$ be arbitrarily fixed time, and let $t$ varies in the bounded interval $[0, T]$.

Then, from (2.4),

$$
u_{i}(t)=e^{-\int_{0}^{t}\left\{\gamma\left(v_{i}\right)+f\right\} d s} u_{0 i}+\beta \delta \int_{0}^{t} e^{-\int_{\tau}^{t}\left\{\gamma\left(v_{i}\right)+f\right\} d s} w_{i}(\tau) d \tau, \quad i=1,2 .
$$

Consequently,

$$
\begin{aligned}
u_{2}(t)-u_{1}(t)= & e^{-\int_{0}^{t}\left\{\gamma\left(v_{1}\right)+f\right\} d s}\left(e^{-\int_{0}^{t}\left\{\gamma\left(v_{2}\right)-\gamma\left(v_{1}\right)\right\} d s}-1\right) u_{01} \\
& +e^{-\int_{0}^{t}\left\{\gamma\left(v_{2}\right)+f\right\} d s}\left(u_{02}-u_{01}\right)+\beta \delta \int_{0}^{t} e^{-\int_{\tau}^{t}\left\{\gamma\left(v_{2}\right)+f\right\} d s}\left(w_{2}(\tau)-w_{1}(\tau)\right) d \tau \\
& +\beta \delta \int_{0}^{t} e^{-\int_{\tau}^{t}\left\{\gamma\left(v_{1}\right)+f\right\} d s}\left(e^{-\int_{\tau}^{t}\left\{\gamma\left(v_{2}\right)-\gamma\left(v_{1}\right)\right\} d s}-1\right) w_{1}(\tau) d \tau .
\end{aligned}
$$

In view of (2.7), (2.8) and (2.10), we obtain that

$$
\begin{aligned}
\left\|u_{2}(t)-u_{1}(t)\right\|_{L^{2}} \leq & \left\|u_{02}-u_{01}\right\|_{L^{2}}+C p\left(\left\|U_{01}\right\|_{X}+\left\|U_{02}\right\|_{X}\right) \\
& \times\left\{\left\|e^{-\int_{0}^{t}\left\{\gamma\left(v_{2}\right)-\gamma\left(v_{1}\right)\right\} d s}-1\right\|_{L^{2}}+\int_{0}^{t}\left\|w_{2}(\tau)-w_{1}(\tau)\right\|_{L^{2}} d \tau\right. \\
& \left.+\int_{0}^{t}\left\|e^{-\int_{\tau}^{t}\left\{\gamma\left(v_{2}\right)-\gamma\left(v_{1}\right)\right\} d s}-1\right\|_{L^{2}}\left(1+\tau^{-\eta}\right) d \tau\right\}, \quad 0 \leq t \leq T .
\end{aligned}
$$

For any $R>0$, there exists a constant $C_{R}>0$ such that $\left|e^{\xi}-1\right| \leq C_{R}|\xi|$ holds for all $|\xi| \leq R$. Using this estimate, we verify that 


$$
\left\|e^{-\int_{0}^{t}\left\{\gamma\left(v_{2}\right)-\gamma\left(v_{1}\right)\right\} d s}-1\right\|_{L^{2}} \leq C p\left(\left\|U_{01}\right\|_{X}+\left\|U_{02}\right\|_{X}\right) \int_{0}^{t}\left\|v_{2}(\tau)-v_{1}(\tau)\right\|_{L^{2}} d \tau .
$$

Similarly,

$$
\begin{aligned}
& \int_{0}^{t}\left\|e^{-\int_{\tau}^{t}\left\{\gamma\left(v_{2}\right)-\gamma\left(v_{1}\right)\right\} d s}-1\right\|_{L^{2}} \tau^{-(1+\varepsilon) / 2} d \tau \\
& \quad \leq C p\left(\left\|U_{01}\right\|_{X}+\left\|U_{02}\right\|_{X}\right) \int_{0}^{t} \int_{\tau}^{t}\left\|v_{2}(s)-v_{1}(s)\right\|_{L^{2}} \tau^{-(1+\varepsilon) / 2} d s d \tau \\
& \quad \leq C p\left(\left\|U_{01}\right\|_{X}+\left\|U_{02}\right\|_{X}\right) \int_{0}^{t}\left\|v_{2}(s)-v_{1}(s)\right\|_{L^{2}} d s
\end{aligned}
$$

Hence,

$$
\begin{aligned}
& \left\|u_{2}(t)-u_{1}(t)\right\|_{L^{2}} \leq\left\|u_{02}-u_{01}\right\|_{L^{2}}+C p\left(\left\|U_{01}\right\|_{X}+\left\|U_{02}\right\|_{X}\right) \\
& \quad \times \int_{0}^{t}\left\{\left\|v_{2}(\tau)-v_{1}(\tau)\right\|_{L^{2}}+\left\|w_{2}(\tau)-w_{1}(\tau)\right\|_{L^{2}}\right\} d \tau, \quad 0 \leq t \leq T .
\end{aligned}
$$

In a similar way, from (2.5) it follows that

$$
\begin{aligned}
\left\|v_{2}(t)-v_{1}(t)\right\|_{L^{2}} \leq & \left\|v_{02}-v_{01}\right\|_{L^{2}} \\
& +C \int_{0}^{t}\left\|u_{2}(\tau)-u_{1}(\tau)\right\|_{L^{2}} d \tau, \quad 0 \leq t \leq T .
\end{aligned}
$$

Finally, from (2.6) we have

$$
w_{2}(t)-w_{1}(t)=e^{-t \Lambda}\left(w_{02}-w_{01}\right)+\alpha \int_{0}^{t} e^{-(t-\tau) \Lambda}\left\{v_{2}(\tau)-v_{1}(\tau)\right\} d \tau .
$$

Therefore,

$$
\begin{aligned}
\left\|w_{2}(t)-w_{1}(t)\right\|_{L^{2}} \leq & \left\|w_{02}-w_{01}\right\|_{L^{2}} \\
& +\alpha \int_{0}^{t}\left\|v_{2}(\tau)-v_{1}(\tau)\right\|_{L^{2}} d \tau, \quad 0 \leq t \leq T .
\end{aligned}
$$

Summing up (5.1), (5.2) and (5.3) and using Gronwall's inequality, we conclude that

$$
\begin{gathered}
\left\|u_{2}(t)-u_{1}(t)\right\|_{L^{2}}+\left\|v_{2}(t)-v_{1}(t)\right\|_{L^{2}}+\left\|w_{2}(t)-w_{1}(t)\right\|_{L^{2}} \\
\leq\left\|U_{02}-U_{01}\right\|_{L^{2}} e^{C p\left(\left\|U_{01}\right\|_{X}+\left\|U_{02}\right\|_{X}\right) t}, \quad 0 \leq t \leq T .
\end{gathered}
$$

This shows that, for $0 \leq t \leq T$, the semigroup $S(t)$ is continuous in the $L^{2}$ topology. But, as $T>0$ is arbitrary, it is the same for any $0 \leq t<\infty$. 
It is now immediate to prove the assertion of proposition. Let $\bar{U} \in$ $L^{2}-\omega\left(U_{0}\right)$. By definition there exists a sequence $t_{n}$ tending to $\infty$ such that $S\left(t_{n}\right) U_{0} \rightarrow \bar{U}$ in the $L^{2}$ topology. By the $L^{2}$ continuity proved above, we have $S\left(t_{n}+t\right) U_{0}=S(t) S\left(t_{n}\right) U_{0} \rightarrow S(t) \bar{U}$ in $L^{2}$. Therefore, $S(t) \bar{U} \in L^{2}-\omega\left(U_{0}\right)$.

Theorem 5.2. For any $U_{0} \in K, L^{2}-\omega\left(U_{0}\right)$ consists of equilibria of the dynamical system.

Proof. Let $\bar{U}=(\bar{u}, \bar{v}, \bar{w}) \in L^{2}-\omega\left(U_{0}\right)$. There exists a sequence $t_{n} \rightarrow \infty$ such that $S\left(t_{n}\right) U_{0}=U\left(t_{n}\right) \rightarrow \bar{U}$ in the $L^{2}$ topology. Since $w\left(t_{n}\right)$ is a bounded sequence in $H^{2}(\Omega)$, we can take a subsequence $\left\{w\left(t_{n^{\prime}}\right)\right\}$ of $\left\{w\left(t_{n}\right)\right\}$ such that $w\left(t_{n^{\prime}}\right) \rightarrow \bar{w}^{\prime}$ strongly in $H^{1}(\Omega)$. It is then easy to see that $\bar{w}=\bar{w}^{\prime}$. Meanwhile, in view of (2.7) and (2.8), $u\left(t_{n}\right) \rightarrow \bar{u}$ and $v\left(t_{n}\right) \rightarrow \bar{v}$ in any $L^{p}$ topology with finite $p$ such that $2 \leq p<\infty$.

By these facts we conclude that the Lyapunov function $\Psi\left(U\left(t_{n^{\prime}}\right)\right)$ given by (3.4) is convergent to $\Psi(\bar{U})$ as $t_{n^{\prime}} \rightarrow \infty$. That is,

$$
\Psi(\bar{U})=\lim _{n^{\prime} \rightarrow \infty} \Psi\left(U\left(t_{n^{\prime}}\right)\right)=\inf _{0 \leq t<\infty} \Psi\left(S(t) U_{0}\right) \equiv \Psi_{\infty}
$$

This means that $\Psi(\bar{U}) \equiv \Psi_{\infty}$ for all $\bar{U}$ 's of vectors in $L^{2}-\omega\left(U_{0}\right)$. By Proposition 5.1, $S(t) \bar{U} \in L^{2}-\omega\left(U_{0}\right)$ for every $t>0$. Hence,

$$
\Psi(S(t) \bar{U}) \equiv \Psi_{\infty}, \quad 0<t<\infty, \bar{U} \in L^{2}-\omega\left(U_{0}\right) .
$$

Furthermore, let $S(t) \bar{U}=\bar{U}(t)=(\bar{u}(t), \bar{v}(t), \bar{w}(t))$; then, by (3.3), we have

$$
\frac{d}{d t} \Psi(\bar{U}(t))=-\int_{\Omega}\left[\alpha\{\gamma(\bar{v})+f+h\}\left(\frac{\partial \bar{v}}{\partial t}\right)^{2}+f \beta \delta\left(\frac{\partial \bar{w}}{\partial t}\right)^{2}\right] d x \equiv 0, \quad 0<t<\infty .
$$

Hence, $(\partial \bar{v} / \partial t)(t) \equiv(\partial \bar{w} / \partial t)(t) \equiv 0$ for $0<t<\infty$. In addition, from the second equation of (2.1), it follows that $f \bar{u}(t) \equiv h \bar{v}(t)$; hence, $(\partial \bar{u} / \partial t)(t) \equiv 0$ for $0<t<\infty$. Thus it has been shown that $S(t) \bar{U} \equiv \bar{U}$ for every $0<t<\infty$, namely, $\bar{U}$ must be an equilibrium.

\section{Numerical examples}

In this section, we will present some numerical example of solution to the problem (1.1) which shows that, even if initial functions $u_{0}, v_{0}$ and $w_{0}$ are continuous, the solution $(u(t), v(t), w(t))$ of (1.1) can tend to a discontinuous stationary solution $(\bar{u}, \bar{v}, \bar{w})$ as $t \rightarrow \infty, \bar{u}$ and $\bar{v}$ being discontinuous and $\bar{w}$ being continuous in $\Omega$.

We consider the following problem 


$$
\begin{cases}\frac{\partial u}{\partial t}=w-\left\{(v-3)^{2}+0.2\right\} u-u & \text { in } \Omega \times(0, \infty), \\ \frac{\partial v}{\partial t}=u-0.45 v & \text { in } \Omega \times(0, \infty), \\ \frac{\partial w}{\partial t}=0.05 \Delta w-w+v & \text { in } \Omega \times(0, \infty), \\ \frac{\partial w}{\partial n}=0 & \text { on } \partial \Omega \times(0, \infty), \\ u(x, y, 0)=u_{0}(x, y), v(x, y, 0)=v_{0}(x, y), & \\ w(x, y, 0)=w_{0}(x, y) & \text { in } \Omega,\end{cases}
$$

where $\Omega=\{(x, y) ; 0<x<5,0<y<5\}$ is a quadratic domain. The initial functions are chosen as in Figure 1; each function has its maximum at $(2.5,2.5)$ and is radially symmetry at the central point $(2.5,2.5)$; and, $v_{0}$ and $w_{0}$ coincide.

After $t=10$, the graph of solution $(u, v, w)$ is stabilized and the Lyapunov function (3.4) also keeps invariance in its values. The figure 2 shows the graph of solution at $t=4000$. Numerically, this solution can be regarded as a stationary solution of $(6.1)$.

The figure 3 shows the graph of initial functions and that of solution at $t=4000$ in the cross-section by a plane which is orthogonal to the $x-y$ plane

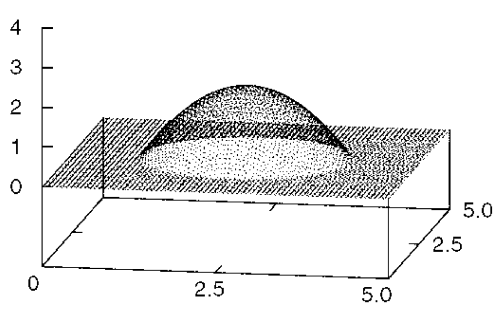

(a) Graph of $u_{0}$

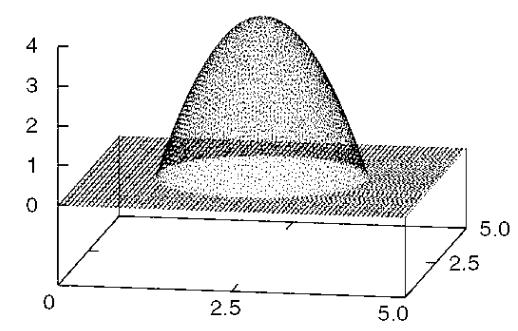

(b) Graph of $v_{0}$

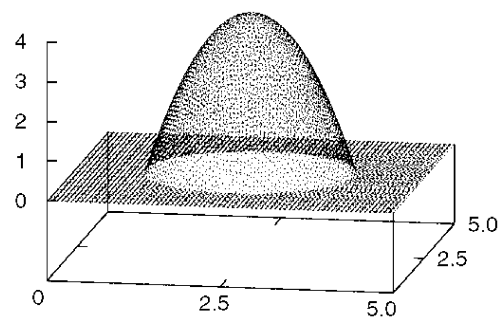

(c) Graph of $w_{0}$

Fig. 1. Initial functions 


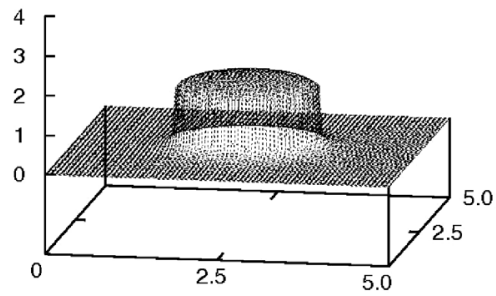

(a) Graph of $u$

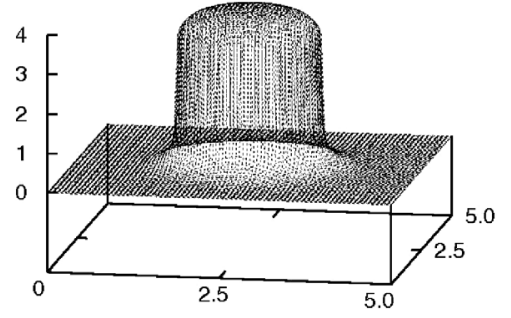

(b) Graph of $v$

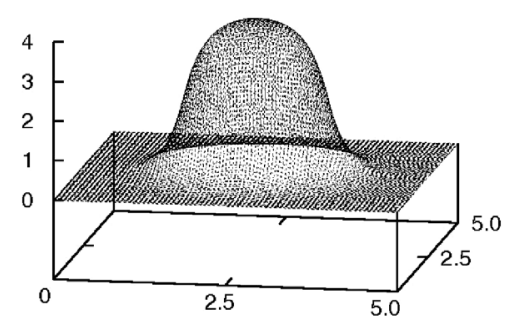

(c) Graph of $w$

Fig. 2. Solution at $t=4000$

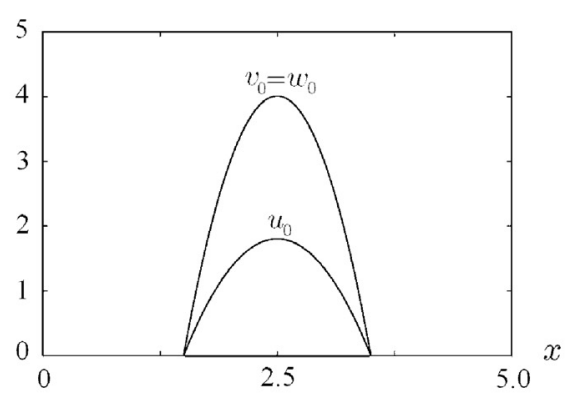

(a) Graph of initial functions

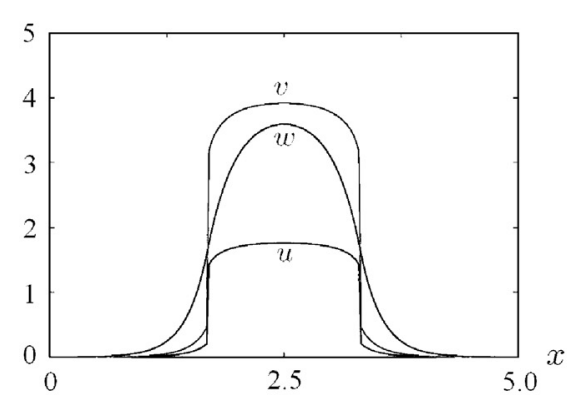

(b) Graph of solution at $t=4000$

Fig. 3. Cross-section by a plane

and contains a line $y=5 / 2$ in the $x-y$ plane. As the figures show, the graphs of $u$ and $v$ of solution have clear discontinuous gaps.

\section{Appendix}

Let us consider the initial-boundary value problem for the FitzhughNagumo equations 


$$
\begin{cases}\frac{\partial u}{\partial t}=d \Delta u+f(u)-v & \text { in } \Omega \times(0, \infty), \\ \frac{\partial v}{\partial t}=b u-\gamma v & \text { in } \Omega \times(0, \infty), \\ \frac{\partial u}{\partial n}=0 & \text { on } \partial \Omega \times(0, \infty), \\ u(x, 0)=u_{0}(x), v(x, 0)=v_{0}(x) & \text { in } \Omega\end{cases}
$$

(cf. $[14,(1.94)])$ in a convex or $\mathscr{C}^{2}$, bounded domain $\Omega$ in $\boldsymbol{R}^{2}$. Here, $f(u)=u(a-u)(u-1)$ is a cubic function with $0<a<1$; and, $b$ and $\gamma$ are positive constants. We set the underlying product space $X$ by

$$
X=\left\{\left(\begin{array}{l}
u \\
v
\end{array}\right) ; u \in L^{2}(\Omega) \text { and } v \in L^{\infty}(\Omega)\right\}
$$

and the space $K$ of initial values by

$$
K=\left\{U_{0}=\left(\begin{array}{c}
u_{0} \\
v_{0}
\end{array}\right) ; u_{0} \in H^{1 / 2}(\Omega ; \boldsymbol{R}) \text { and } v_{0} \in L^{\infty}(\Omega ; \boldsymbol{R})\right\} .
$$

In $X,(7.1)$ is formulated as the following Cauchy problem

$$
\left\{\begin{array}{l}
\frac{d U}{d t}+A U=F(U), \quad 0<t<\infty \\
U(0)=U_{0} \in K
\end{array}\right.
$$

Here, $A$ is a sectorial linear operator of $X$ given by

$$
A=\left(\begin{array}{ll}
\Lambda & 0 \\
0 & \gamma
\end{array}\right) \quad \text { with } \quad \mathscr{D}(A)=\left\{\left(\begin{array}{l}
u \\
v
\end{array}\right) ; u \in H_{N}^{2}(\Omega) \text { and } v \in L^{\infty}(\Omega)\right\},
$$

where $\Lambda$ is a realization of the operator $-d \Delta+1$ in $L^{2}(\Omega)$ under the homogeneous Neumann boundary conditions on the boundary $\partial \Omega$ and is a positive definite self-adjoint operator of $L^{2}(\Omega)$ with $\mathscr{D}(\Lambda)=H_{N}^{2}(\Omega)$. Then, $U_{0} \in K$ if and only if $U_{0} \in \mathscr{D}\left(A^{1 / 4}\right)$ and $U_{0}$ is real. The nonlinear operator $F: \mathscr{D}\left(A^{\eta}\right) \rightarrow$ $X$ is given by

$$
F(U)=\left(\begin{array}{c}
u+f(u)-v \\
b u
\end{array}\right), \quad U=\left(\begin{array}{l}
u \\
v
\end{array}\right) \in \mathscr{D}\left(A^{\eta}\right),
$$

where $\eta$ is some fixed exponent such that $1 / 2<\eta<1$.

It is then possible to apply [1, Theorem 2.1] to (7.2) with $\mu=1 / 4$ and with the $\eta, 1 / 2<\eta<1$, fixed above to construct the global solution (cf. Section 2). As the result, for any $U_{0} \in K$, there exists a unique global solution to (7.2) in the function space 


$$
U \in \mathscr{C}\left([0, \infty) ; \mathscr{D}\left(A^{1 / 4}\right)\right) \cap \mathscr{C}((0, \infty) ; \mathscr{D}(A)) \cap \mathscr{C}^{1}((0, \infty) ; X)
$$

with values in $K$. Furthermore, we can establish the global estimates of solutions

$$
\begin{gathered}
\left\|A^{1 / 2} U(t)\right\|_{X} \leq C\left\{\left(t^{-1 / 4}+1\right) e^{-\delta t}\left\|A^{1 / 4} U_{0}\right\|_{X}+1\right\}, \quad 0 \leq t<\infty, \\
\|A U(t)\|_{X} \leq\left(t^{-3 / 4}+1\right) p\left(\left\|A^{1 / 4} U_{0}\right\|_{X}\right), \quad 0<t<\infty,
\end{gathered}
$$

where $\delta>0$ is some positive exponent and $p(\cdot)$ is some continuous increasing function. It is also verified that the solution is Lipschitz continuous with respect to the initial value, namely, $\|U(t)-V(t)\|_{X} \leq L_{T}\left\|U_{0}-V_{0}\right\|_{X}$ for $U_{0}, V_{0} \in K$ and $t \in[0, T], T>0$ being any fixed time.

So, we can define a dynamical system $(S(t), K, X)$ from (7.2). For $S(t) U_{0}=U(t)$, we have

$$
v(t)=e^{-\gamma t} v_{0}+b \int_{0}^{t} e^{-\gamma(t-s)} u(s) d s, \quad 0<t<\infty .
$$

It is clear that $e^{-\gamma t} v_{0} \rightarrow 0$ in $L^{\infty}(\Omega)$ as $t \rightarrow \infty$. Meanwhile,

$$
\left\|\int_{0}^{t} e^{-\gamma(t-s)} u(s) d s\right\|_{H^{2 \eta}} \leq \int_{0}^{t} e^{-\gamma(t-s)}\|u(s)\|_{H^{2 \eta}} d s
$$

and, from (7.3) and (7.4),

$$
\begin{aligned}
\|u(s)\|_{H^{2 \eta}} & \leq\left\|A^{\eta} U(s)\right\|_{X} \leq C\|A U(s)\|_{X}^{2 \eta-1}\left\|A^{1 / 2} U(s)\right\|_{X}^{2(1-\eta)} \\
& \leq\left(s^{1 / 4-\eta}+1\right) p_{\eta}\left(\left\|A^{1 / 4} U_{0}\right\|_{X}\right)
\end{aligned}
$$

with some continuous increasing function $p_{\eta}(\cdot)$. Therefore,

$$
\begin{aligned}
\int_{0}^{t / 2} e^{-\gamma(t-s)}\|u(s)\|_{H^{2 \eta}} d s & \leq e^{-\gamma t / 2} \int_{0}^{t / 2} e^{-\gamma(t / 2-s)}\left(s^{1 / 4-\eta}+1\right) d s p_{\eta}\left(\left\|A^{1 / 4} U_{0}\right\|_{X}\right) \\
& \leq C e^{-\gamma t / 2} p_{\eta}\left(\left\|A^{1 / 4} U_{0}\right\|_{X}\right),
\end{aligned}
$$

and

$$
\begin{aligned}
\int_{t / 2}^{t} e^{-\gamma(t-s)}\|u(s)\|_{H^{2 \eta}} d s & \leq \int_{t / 2}^{t} e^{-\gamma(t-s)}\left((s-t / 2)^{1 / 4-\eta}+1\right) d s p_{\eta}\left(\left\|A^{1 / 4} U(t / 2)\right\|_{X}\right) \\
& \leq C p_{\eta}\left(C\left\{\left(2^{1 / 4} t^{-1 / 4}+1\right) e^{-\delta t / 2}\left\|A^{1 / 4} U_{0}\right\|_{X}+1\right\}\right) .
\end{aligned}
$$

Hence, there exists a constant $\tilde{C}>0$ for which the following assertion is valid. For any $R>0$, there exists a time $T_{R}>0$ such that 


$$
\int_{0}^{t} e^{-\gamma(t-s)}\|u(s) d s\|_{H^{2 \eta}} d s \leq \tilde{C}, \quad \forall U_{0} \in K \cap B^{X}(0 ; R), \forall t \geq T_{R} .
$$

In particular, on account of the compact embedding $\mathscr{D}\left(A^{\eta}\right) \subset L^{\infty}(\Omega)$, this implies that the $\omega$-limit set $\omega\left(U_{0}\right)$ of the trajectory $S(t) U_{0}$ is nonempty for every $U_{0} \in K$. Moreover, it is possible to construct the global attractor $\mathscr{A}$ for the dynamical system $(S(t), K, X)$.

\section{References}

[1] Chuan, L. H. and Yagi, A., Dynamical system for forest kinematic model, Adv. Math. Sci. Appl., 16 (2006), to appear.

[2] Chuan, L. H., Tsujikawa, T. and Yagi, A., Structure of stationary solutions for forest kinematic model, submitted.

[3] Kuznetsov, Yu. A., Antonovsky, M. Ya., Biktashev, V. N. and Aponina, A., A crossdiffusion model of forest boundary dynamics, J. Math. Biol., 32 (1994), 219-232.

[4] Lin, Y. and Liu, Y., Qualitative analysis for a model of forest with diffusion and nonlocal effects, Nonlinear Analysis, 39 (2000), 217-229.

[5] Nakaguchi, E. and Yagi, A., Fully discrete approximation by Galerkin Runge-Kutta methods for quasilinear parabolic systems, Hokkaido Math. J., 31 (2002), 385-429.

[6] Nakata, H., Numerical simulations for forest boundary dynamics model, Master's thesis, Osaka University (2004).

[7] Osaki, K. and Yagi, A., Global existence for a chemotaxis-growth system in $\boldsymbol{R}^{2}$, Adv. Math. Sci. Appl., 12 (2002), 587-606.

[ 8 ] Wu, Y., Stability of travelling waves for a cross-diffusion model, J. Math. Anal. Appli., 215 (1997), 388-414.

[9] Wu, Y. and Lin, Y., The stability of steady states for a model with diffusion and spatial average, J. Math. Anal. Appli., 232 (1999), 259-271.

[10] Babin, A. V. and Vishik, M. I., Attractors of Evolution Equations, North-Holland, Amsterdam, 1992.

[11] Dautray, R. and Lions, J. L., Mathematical analysis and numerical methods for science and technology, Vol. 2, Springer-Verlag, Berlin, 1988.

[12] Grisvard, P., Elliptic Problems in Nonsmooth Domains, Pitman, London, 1985.

[13] Sell, G. R. and You, Y., Dynamics of Evolutionary Equations, Springer, New York, 2002.

[14] Murray, J. D., Mathematical Biology, II: Spacial Models and Biomedical Applications, Springer, New York, 2003.

[15] Temam, R., Infinite-Dimensional Dynamical Systems in Mechanics and Physics, 2nd ed., Springer, New York, 1997.

[16] Triebel, H., Interpolation theory, function spaces, differential operators, North-Holland, Amsterdam, 1978.

\author{
nuna adreso: \\ Le Huy Chuan \\ Department of Environmental Technology \\ Osaka University \\ Suita, Osaka 565-0871 \\ Japan \\ E-mail: chuanlh@ap.eng.osaka-u.ac.jp
}


Tohru Tsujikawa

Faculty of Engineering

Miyazaki University

Miyazaki 889-2192

Japan

E-mail: tujikawa@cc.miyazaki-u.ac.jp

Atsushi Yagi

Department of Applied Physics

Osaka University

Suita, Osaka 565-0871

Japan

E-mail: yagi@ap.eng.osaka-u.ac.jp

(Ricevita la 29-an de novembro, 2005) 